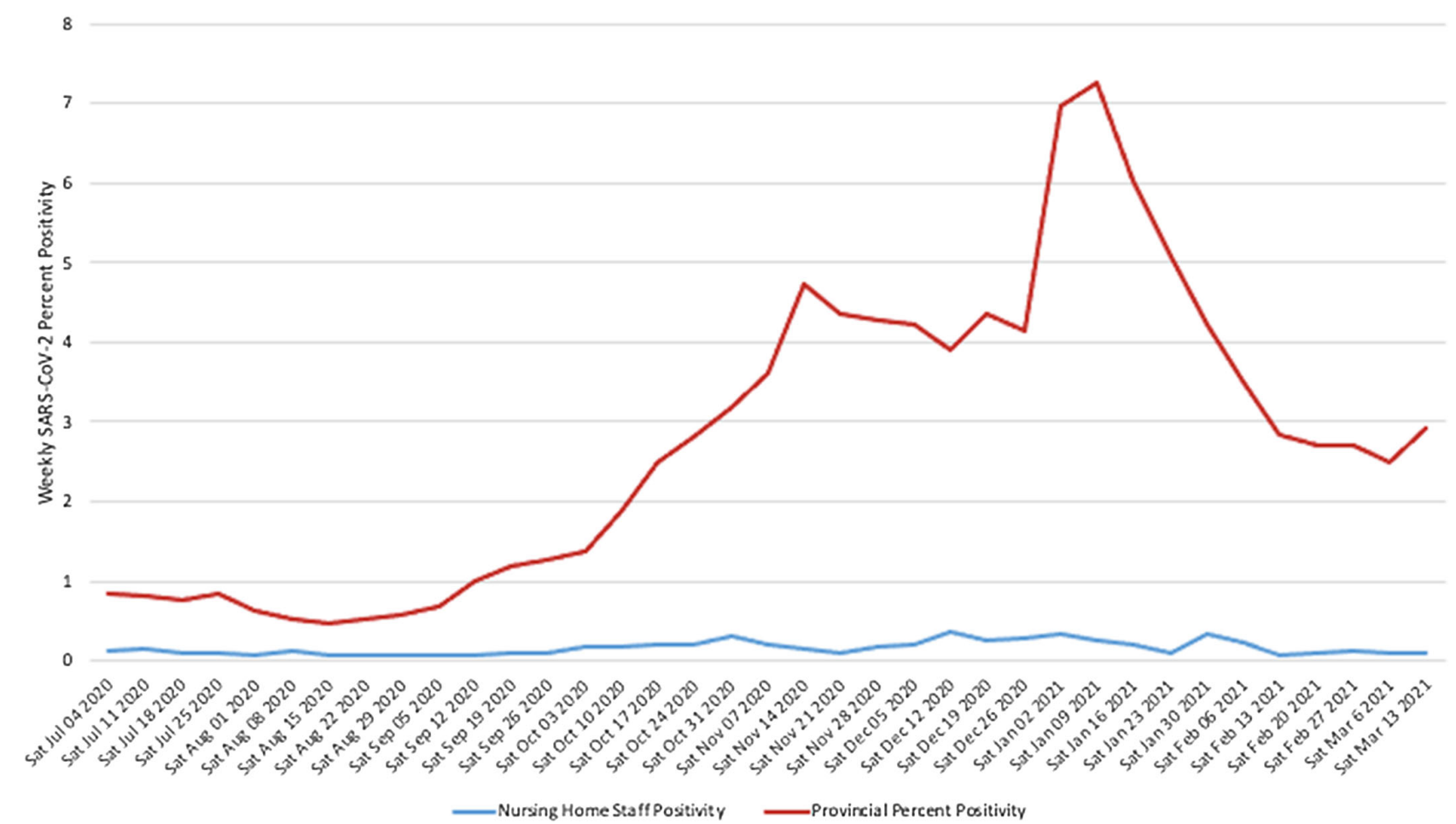

Fig. 1. Ontario nursing-home staff screen testing SARS-CoV-2 percent positivity compared to provincial percent positivity, from June 28,2020 , to March 13 , 2021. Line graph showing percent SARS-CoV-2 test positivity for routine asymptomatic nursing-home staff screening compared to provincial test positivity between June 28,2020 , and March 13 , 2021. During the low SARS-CoV-2 incidence summer months of 2020, test positivity rates were consistently $<0.1 \%$. There was a small increase in SARS-CoV-2 test positivity during the second wave (September 1, 2020-March 14, 2021) peaking at 0.36\% in December 2020.

2. Snoeck CJ, Vaillant M, Abdelrahman T, et al. Prevalence of SARS-CoV-2 infection in the Luxembourgish population: the CON-VINCE study. medRxiv 2020. doi: 10.1101/2020.05.11.20092916.

3. Olalla J, Corrrea AM, Martin-Escalante MC, et al. Search for asymptomatic carriers of SARS-CoV-2 in healthcare workers during the pandemic: a Spanish experience. QJM 2020;113:794-798.

4. Steele R. Updated long-term care sector surveillance testing strategy. Ontario Ministry of Long-Term Care website. https://ohwestcovid19.ca/wp-content/ uploads/2020/11/20201124-DM-Memo-Updated-Long-Term-Care-Sector-
Surveillance-Testing-Strategy-Nov-22-2020.pdf. Published online November 22, 2020. Accessed March 15, 2021.

5. Sutton D, Fuchs K, D'Alton M and Goffman D. Universal screening for SARS-CoV-2 in women admitted for delivery. N Engl J Med 2020;382: 2163-2164.

6. Brown K, Stall NM, Vanniyasingam $\mathrm{T}$, et al. Early impact of Ontario's COVID-19 vaccine rollout on long-term care home residents and health care workers. Ontario Science Table 2021. doi: 10.47326/ocsat. 2021.02.13.1.0.

\title{
Rostered routine testing for severe acute respiratory coronavirus virus 2 (SARS-CoV-2) infection among healthcare personnel-Is there a role in a tertiary-care hospital with enhanced infection prevention and control measures and robust sickness-surveillance systems?
}

\author{
Angela Chow PhD ${ }^{1,2,3}$ (D) , Huiling Guo MPH${ }^{1}$, Win Mar Kyaw MPH${ }^{1}$, Anthony Lianjie Li MD ${ }^{1}$, Rachel Hui Fen Lim MPH ${ }^{1}$ and \\ Brenda Ang $\mathrm{MPH}^{4,5}$ \\ ${ }^{1}$ Department of Clinical Epidemiology, Office of Clinical Epidemiology, Analytics, and Knowledge, Tan Tock Seng Hospital, Singapore, ${ }^{2}$ Saw Swee Hock School of \\ Public Health, National University of Singapore, ${ }^{3}$ Lee Kong Chian School of Medicine, Nanyang Technological University, Singapore, ${ }^{4}$ Department of Infectious \\ Disease, Tan Tock Seng Hospital, Singapore and ${ }^{5}$ Department of Infection Prevention and Control, Tan Tock Seng Hospital, Singapore
}

Author for correspondence: Angela Chow, E-mail: angela_chow@ttsh.com.sg Cite this article: Chow A, et al. (2022). Rostered routine testing for severe acute respiratory coronavirus virus 2 (SARS-CoV-2) infection among healthcare personnelIs there a role in a tertiary-care hospital with enhanced infection prevention and control measures and robust sickness-surveillance systems?. Infection Control \& Hospital Epidemiology, 43: 1528-1530, https://doi.org/10.1017/ice.2021.268
To the Editor-Active surveillance allows (1) early identification and isolation of individuals infected with severe acute respiratory coronavirus virus 2 (SARS-CoV-2), (2) tracing and quarantining close contacts, and (3) prevention of further transmission. 
Table 1. Categories of Healthcare Personnel (HCP) Screened for and Detected With SARS-CoV-2 Infection, April 28-May 22, 2021

\begin{tabular}{|c|c|c|c|c|c|}
\hline \multirow[b]{2}{*}{ HCP Category } & \multirow[b]{2}{*}{ Screened, No. } & \multicolumn{3}{|c|}{ SARS-CoV-2 PCR Test Result } & \multirow[b]{2}{*}{$\begin{array}{l}\text { SARS-CoV-2 Detection } \\
\text { Rate, } \%\end{array}$} \\
\hline & & Detected, No. & $\begin{array}{l}\text { Equivocal But Not Detected On } 2 \text { Subsequent Samples } \\
\text { Sent } 24 \text { h Apart, No. }\end{array}$ & $\begin{array}{c}\text { Not } \\
\text { Detected, No. }\end{array}$ & \\
\hline Close contact $^{\mathrm{a}}$ & 416 & 3 & 0 & 413 & 0.7 \\
\hline Visited affected ward ${ }^{b}$ & 634 & 1 & 0 & 633 & 0.2 \\
\hline Other HCP & 11,004 & 0 & 10 & 10,994 & 0 \\
\hline
\end{tabular}

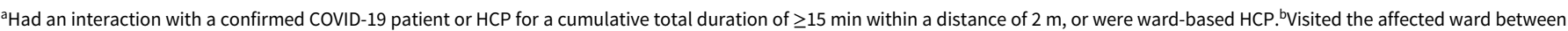
April 20 and 28, 2021, for a cumulative total duration of $\geq 15 \mathrm{~min}$.

Simulation studies suggest that rostered routine testing (RRT) for asymptomatic healthcare personnel (HCP) amid ongoing community transmission can substantially reduce the risk of coronavirus disease 2019 (COVID-19) outbreaks in hospitals. ${ }^{1}$

With the low prevalence of SARS-CoV-2 in asymptomatic HCP of $0.1 \%-0.4 \%$, the benefits of RRT for asymptomatic HCP in hospitals with good infection prevention and control practices and robust staff acute respiratory illness (ARI) surveillance systems remain questionable. ${ }^{2-7}$ However, with the emergence of more highly transmissible SARS-CoV-2 variant strains and institutional outbreaks caused by them, ${ }^{8}$ Singapore's Ministry of Health has implemented RRT for HCP working in acute-care hospitals.

On April 28, 2021, a nurse working in a general ward in Tan Tock Seng Hospital (TTSH) was confirmed with COVID-19 after seeking medical attention for ARI. A patient receiving care in the same ward was also confirmed with COVID-19 later that day. By May 22, 47 COVID-19 cases had been linked to the ward cluster caused by the B.1.617.2 variant strain. We describe the experience at TTSH in detecting COVID-19 in (1) HCP who were close contacts of COVID-19 cases linked to the cluster, (2) HCP who had been to the affected ward, and (3) asymptomatic HCP screened as part of outbreak management.

The TTSH is a 1,600-bed acute tertiary-care hospital with mostly multibed rooms with 4-6 patients each. The affected location was a multidisciplinary general ward without special isolation facilities. Aside from nurses and housekeepers who were wardbased, other HCP including physicians, surgeons, pharmacists, therapists, phlebotomists, and porters moved between wards.

Upon detection of the cluster on April 28, 2021, the affected ward was locked down and contact tracing was initiated. HCP close contacts were defined as those who had interacted with a confirmed COVID-19 patient or HCP for a cumulative duration of $\geq 15$ minutes within a distance of $2 \mathrm{~m}$, or ward-based HCP. Close contacts were placed on quarantine and were screened for SARS-CoV-2 infection via polymerase chain reaction (PCR) test on entry to quarantine and at 7, 14, and 21 days from the date of last exposure to the confirmed case or to the ward. Furthermore, HCP who had visited the affected ward between April 20 and 28, 2021, for a cumulative duration of $\geq 15$ minutes were also identified, placed on quarantine, and screened for SARS-CoV-2 infection on entry to quarantine and at 7,14 , and 21 days from the date of last exposure to the affected ward. The first patient identified with COVID-19 was admitted to the ward on April 20, and the ward was locked down on April 28. Additionally, all asymptomatic HCP working in the hospital underwent weekly SARS-CoV-2 PCR testing until the affected ward reopened on May 22.

Among $416 \mathrm{HCP}$ close contacts who were placed under quarantine, $1 \mathrm{HCP}$ was detected with SARS-CoV-2 infection via quarantine on-entry test 1 day prior to ARI symptom onset, and 2 were detected when they developed ARI symptoms within first 4 days of quarantine (Table 1). Among the $634 \mathrm{HCP}$ who had visited the affected ward for $\geq 15$ minutes, $1 \mathrm{HCP}$ was positive 2 days prior to symptom onset.

Of 11,004 asymptomatic staff who had undergone 2 rounds of weekly SARS-CoV-2 PCR testing, none was confirmed with COVID-19. However, 10 were identified to have equivocal results, with high cycle threshold values ranging from 37.42 to 43.30 . For these HCP with equivocal results, subsequent 2 swabs taken 24 hours apart yielded negative results by the hospital's laboratory and the national reference laboratory. All except $1 \mathrm{HCP}$ had nonreactive serology test results; that $\mathrm{HCP}$ was a returned traveler from India who was diagnosed with COVID-19 on arrival to Singapore in March 2021. Each HCP with an equivocal test result was placed on leave of absence (LOA) and was advised to self-isolate at home except for returning to the hospital for tests. Coworkers identified to be close contacts of the HCP were also placed on LOA until the repeated tests returned negative. An average of $7 \mathrm{HCP}$ close contacts (maximum, 18) per HCP with equivocal test results were placed on LOA from work for a mean duration of 3.5 days (maximum, 5).

Although the screening of presymptomatic and symptomatic HCP close contacts and HCP who had visited the affected ward yielded a SARS-CoV-2 detection rate of $0.7 \%$ and $0.2 \%$ respectively, hospital-wide weekly screening of other asymptomatic HCP did not detect any SARS-CoV-2 infections. Instead, the hospital-wide screening resulted in a loss of productivity of $292 \mathrm{HCP}-$ workdays, with a mean of 3.5 work days lost per HCP placed on LOA. For the HCP (a cook in the hospital) with the greatest number of HCP close contacts $(n=18)$ placed on LOA, response to the equivocal test result caused a reduction in food choices and compromised the nutritional services available to patients.

Even in the wake of a ward cluster due to a highly transmissible SARS-CoV-2 variant strain, the extensive hospital-wide testing of asymptomatic HCP did not uncover any covert infections. With the hospital's robust infection prevention and control measures and HCP ARI surveillance system, the implementation of RRT may yield limited benefits and paradoxically exacerbate strained manpower and laboratory resources that could be conserved to manage community SARS-CoV-2 infections.

Acknowledgments. We thank colleagues from TTSH's human resource (Serene Tan and her team) and hospital operations (Michael Leow and his team), departments that worked tirelessly to collate the data on HCP on quarantine and weekly SARS-CoV-2 PCR testing.

Financial support. No financial support was provided relevant to this article.

Conflicts of interest. All authors report no conflicts of interest relevant to this article. 


\section{References}

1. Chin ET, Huynh BQ, Chapman LAC, et al. Frequency of routine testing for coronavirus disease 2019 (COVID-19) in high-risk healthcare environments to reduce outbreaks. Clin Infect Dis 2020. doi: 10.1093/cid/ciaa1383.

2. Shenoy ES, Weber DJ. Routine surveillance of asymptomatic healthcare personnel for severe acute respiratory coronavirus virus 2 (SARS-CoV-2): not a prevention strategy. Infect Control Hosp Epidemiol 2021;42:592-597.

3. Chow A, Htun HL, Kyaw WM, Lee LT, Ang B. Asymptomatic health-care worker screening during the COVID-19 pandemic. Lancet 2020;396: 1393-1394.

4. Htun HL, Lim DW, Kyaw WM, et al. Responding to the COVID-19 outbreak in Singapore: staff protection and staff temperature and sickness surveillance systems. Clin Infect Dis 2020;71:1947-1952.
5. Wang Y, Tan JK, Tay MZ, et al. Dancing with COVID-19 after the hammer is lifted: enhancing healthcare worker surveillance. J Infect 2020;81(6):e13-e15.

6. Kyaw WM, Hein AA, Zhang ZX, et al. Healthcare worker acute respiratory illness cluster in 2020: could it be from COVID-19? Infect Control Hosp Epidemiol 2020. doi: 10.1017/ice.2020.364.

7. Lim DW, Htun HL, Wang Y, et al. Healthcare workers as 'canaries' for acute respiratory infections and pathogens during the COVID-19 pandemic. $J$ Hosp Infect 2021;112:119-120.

8. Sim D, Kok X. 'Far from out of the woods': how a COVID-19 variant put Singapore back in defensive mode. South China Morning Post website. https:/www.scmp.com/week-asia/health-environment/article/3134497/farout-woods-how-covid-19-variant-put-singapore-back. Published May 23, 2021. Accessed May 23, 2021.

\title{
Promoting coronavirus disease 2019 (COVID-19) vaccination: Do we need to reframe how we present risk?
}

\author{
Michelle Doll MD, MPH ${ }^{1}$ (]) Jeanine P. D. Guidry $\mathrm{PhD}^{2}$, Rachel Pryor RN, MPH${ }^{1}$, Arthur L. Kellermann MD, MPH ${ }^{1}$ and \\ Michael P. Stevens MD, MPH ${ }^{1}$ \\ ${ }^{1}$ Virginia Commonwealth University Health System, Richmond, Virginia and ${ }^{2}$ Virginia Commonwealth University, Richmond Virginia
}

To the Editor-Although impressive progress has been made in vaccinating the US population against coronavirus disease 2019 (COVID-19), the country has not yet reached the level of uptake needed to assure the added benefit of "herd immunity." 1 Equally if not more concerning, substantial subgroups of the population remain skeptical if not overtly resistant to vaccination. To convince as many of these individuals as possible to change their mind, it is imperative that we communicate the benefits of vaccination in more meaningful ways.

Recently we reported how Virginia Commonwealth University Medical Center in Richmond swiftly contained an outbreak of employee infections that coincided with the "third wave" of COVID-19 that hit Virginia between December 2020 to January 2021. ${ }^{1}$ During a single week (December 13-19), 134 of 13,346 employees, or $\sim 1 \%$ of our entire workforce, became infected with severe acute respiratory coronavirus virus 2 (SARS-CoV-2). Fortunately, we received our first doses of vaccine that same week and began vaccinating employees on December 16. The campaign that followed, along with a bundle of intensified infection-prevention measures, produced a 10 -fold reduction in healthcare worker (HCW) infections (down to $0.1 \%$ ) by January 31). Based on our local experience, we calculated that getting vaccinated against COVID-19 reduces a VCU Health employee's odds of getting infected by $98 \% .^{2}$

A potentially more compelling way to express this beneficial effect is to quantify the relative risk, compared to vaccinated colleagues, that an unvaccinated HCW will become infected with the virus that causes COVID-19. As of May 3, 2021, that risk is 27.9 times greater in unvaccinated HCWs at VCU Health.

\footnotetext{
Author for correspondence: Michelle Doll, E-mail: michelle.doll@vcuhealth.org Cite this article: Doll M, et al. (2022). Promoting coronavirus disease 2019 (COVID-19) vaccination: Do we need to reframe how we present risk?. Infection Control \& Hospital Epidemiology, 43: 1530, https://doi.org/10.1017/ice.2021.302
}

A recent study from Israel reported the real-world effectiveness of the Pfizer-BioNTech mRNA vaccine BNT162b2. The incidence rate of SARS-CoV-2 per 100,000 person days was 91.5 in unvaccinated people versus 3.1 in fully vaccinated people-an effectiveness rate of 95.3 percent. ${ }^{3}$ In other words, unvaccinated people were 29.5 times more likely to become infected than those who were vaccinated.

Rather than focus on the risks of vaccination, which is tiny by comparison, the public would be better served by focusing on the far larger and more severe risk incurred by those who forego or decline repeated opportunities to be vaccinated.

If VCU Health data are representative of the general public, the risk that an unvaccinated adult will become infected with the virus that has killed $>600,000$ Americans and $>3.4$ million people worldwide, is $\sim 28$ times higher than those who accept vaccination. This way of framing risk may be of value to infection prevention specialists involved in COVID-19 vaccine messaging campaigns.

\section{Acknowledgments.}

Financial support. No financial support was provided relevant to this article.

Conflicts of interest. All authors report no conflicts of interest relevant to this article.

\section{References}

1. Randolph HE, Barreiro LB. Herd immunity: understanding COVID-19. Immunity 2020;52:737-741.

2. Pryor R, Cooper K, Britton A, et al. Riding the third wave: how an academic medical center reduced COVID-19 infections in healthcare workers. $N$ Engl J Med Catalyst 2021. doi: 10.1056/CAT.21.0060.

3. Haas EJ, Angulo FJ, McLaughlin JM, et al. Impact and effectiveness of mRNA BNT162b2 vaccine against SARS-CoV-2 infections and COVID-19 cases, hospitalisations, and deaths following a nationwide vaccination campaign in Israel: an observational study using national surveillance data. Lancet 2021;397:1819-1829. 